\title{
Dark Matter and the Energy-Momentum Relationship in a Hydrogen Atom
}

\author{
Koshun Suto \\ Chudai-Ji Temple, Isesaki, Japan \\ Email: Koshun_suto129@mbr.nifty.com
}

How to cite this paper: Suto, K. (2020) Dark Matter and the Energy-Momentum Relationship in a Hydrogen Atom. Journal of High Energy Physics, Gravitation and Cosmology, 6, 52-61. https://doi.org/10.4236/jhepgc.2020.61007

Received: November 14, 2019

Accepted: January 4, 2020

Published: January 7, 2020

Copyright $\odot 2020$ by author(s) and Scientific Research Publishing Inc. This work is licensed under the Creative Commons Attribution International License (CC BY 4.0).

http://creativecommons.org/licenses/by/4.0/

(c) (i) Open Access

\begin{abstract}
Einstein derived the energy-momentum relationship which holds in an isolated system in free space. However, this relationship is not applicable in the space inside a hydrogen atom where there is potential energy. Therefore, in 2011, the author derived an energy-momentum relationship applicable to the electron constituting a hydrogen atom. This paper derives that relationship in a simpler way using another method. From this relationship, it is possible to derive the formula for the energy levels of a hydrogen atom. The energy values obtained from this formula almost match the theoretical values of Bohr. However, the relationship derived by the author includes a state that cannot be predicted with Bohr's theory. In the hydrogen atom, there is an energy level with $n=0$. Also, there are energy levels where the relativistic energy of the electron becomes negative. An electron with this negative energy (mass) exists near the atomic nucleus (proton). The name "dark hydrogen atom" is given to matter formed from one electron with this negative mass and one proton with positive mass. Dark hydrogen atoms, dark hydrogen molecules, other types of dark atoms, and aggregates made up of dark molecules are plausible candidates for dark matter, the mysterious type of matter whose true nature is currently unknown.
\end{abstract}

\section{Keywords}

Einstein's Energy-Momentum Relationship, Classical Quantum Theory, Ultra-Low Energy Levels, Dirac Relativistic Wave Equation, Dark Matter, Dark Hydrogen, Triplet Production

\section{Introduction}

The energy-momentum relationship in the special theory of relativity (STR) holds in an isolated system in free space. Here, if $m_{0}$ is rest mass and $m$ relati- 
vistic mass, the relationship can be written as follows.

$$
\left(m_{0} c^{2}\right)^{2}+p^{2} c^{2}=\left(m c^{2}\right)^{2} \text {. }
$$

However, this relationship is not applicable to the electron in a hydrogen atom where there is potential energy. By all rights, this problem should have been investigated in the first half of the 20th century. However, the problem was thought to have been solved by Dirac's derivation of the relativistic wave equation.

Incidentally, Sommerfeld defined kinetic energy as the energy, a moving object in excess of the stationary object. Therefore, if the rest mass of the object is $m_{0}$ and the mass when moving is $m$, the kinetic energy of the object can be described by the following formula [1].

$$
K=m c^{2}-m_{0} c^{2}=m_{0} c^{2}\left[\frac{1}{\left(1-\beta^{2}\right)^{1 / 2}}-1\right], \quad \beta=\frac{v}{c} .
$$

Here, the following inequality holds.

$$
m_{0}<m
$$

Sommerfeld believed that Equation (2), which can be derived from Equation (1), can also be applied to the electron in a hydrogen atom.

Incidentally, in Bohr's theory, the total mechanical energy $E_{\mathrm{BO}, n}$ of the electron constituting a hydrogen atom is given by the sum of the kinetic energy and potential energy of the electron (Here, $E_{\mathrm{BO}}$ refers to the total mechanical energy predicted by Bohr).

That is,

$$
E_{\mathrm{BO}, n}=K_{n}+V\left(r_{n}\right)
$$

Here, $K$ is the kinetic energy of the entire system, and $V$ is the potential energy of the entire system.

In Equation (4), the energy (i.e., the sum of potential energy and kinetic energy) when the electron is separated from the atomic nucleus (proton) by an infinite distance and placed at rest there, is assumed to be zero. However, according to the STR, when an electron is at rest, it has a rest mass energy of $m_{\mathrm{e}} c^{2}$. From the perspective of the theory of relativity, the relativistic energy $m_{n} c^{2}$ of the electron constituting a hydrogen atom must be defined as follows.

$$
m_{n} c^{2}=m_{\mathrm{e}} c^{2}+E_{n}=m_{\mathrm{e}} c^{2}+K_{n}+V\left(r_{n}\right)
$$

Here, the kinetic energy of a hydrogen atom cannot be described with Equation (2) because $m_{n}<m_{\mathrm{e}}$.

An electron previously at rest emits a photon when it is drawn into the atomic nucleus. At that time, the electron acquires the same amount of kinetic energy as the emitted photonic energy. To ensure that the law of energy conservation holds, some energy must decrease. We look to potential energy as the answer to that problem, but there is no substantiality to potential energy. Thus the author noticed that the potential energy corresponds to the reduction in rest mass 
energy $-\Delta m_{\mathrm{e}} c^{2}$. Also, potential energy was defined as follows [2].

$$
V(r)=-\Delta m_{\mathrm{e}} c^{2} \text {. }
$$

Incidentally, according to the virial theorem, the following relation holds between $K$ and $V$ :

$$
\langle K\rangle=-\frac{1}{2}\langle V\rangle
$$

The average time of $K$ is equal to $-1 / 2$ the time average of $V$. Also, the sum of the time average $K$ and the time average of the total mechanical energy $E$ of the entire system becomes 0 . That is,

$$
\langle K\rangle+\langle E\rangle=0 \text {. }
$$

Next, if Equations (7) and (8) are combined, the result is as follows:

$$
\langle E\rangle=-\langle K\rangle=\frac{1}{2}\langle V\rangle \text {. }
$$

Due to the above, $E_{n}$ was defined as follows:

$$
E_{n}=m_{n} c^{2}-m_{\mathrm{e}} c^{2}, \quad n=1,2, \cdots, E_{n}<0 .
$$

\section{The Relation between Kinetic Energy and Momentum Derived from the STR Relationship}

First, it is clear that the following formula holds.

$$
\left[m_{0} c^{2}+\left(m c^{2}-m_{0} c^{2}\right)\right]^{2}=\left(m c^{2}\right)^{2} \text {. }
$$

Expanding the left side of this equation yields the following.

$$
m_{0}^{2} c^{4}+\left(m^{2} c^{4}-m_{0}^{2} c^{4}\right)=\left(m_{0} c^{2}\right)^{2}+\left(m+m_{0}\right)\left(m c^{2}-m_{0} c^{2}\right) c^{2} .
$$

Using this, Equation (11) becomes as follows.

$$
\left(m_{0} c^{2}\right)^{2}+\left(m+m_{0}\right)\left(m c^{2}-m_{0} c^{2}\right) c^{2}=\left(m c^{2}\right)^{2} .
$$

Since this equation and Equation (1) are equal, the following relationship must hold when Equation (2) is taken into account.

$$
p^{2}=\left(m_{0}+m\right)\left(m c^{2}-m_{0} c^{2}\right)=\left(m_{0}+m\right) K .
$$

The following formula is obtained from this.

$$
K=\frac{p^{2}}{m_{0}+m} .
$$

Equation (15) is the formula for relativistic kinetic energy [3]. Classical (nonrelativistic) kinetic energy, in contrast, is defined as follows.

$$
K=\frac{1}{2} m_{0} v^{2}=\frac{p^{2}}{2 m_{0}} \text {. }
$$

\section{Energy-Momentum Relationship of the Electron Derived with Another Method}

The author has previously derived the following relationships applicable to the 
electron constituting a hydrogen atom [4].

$$
\left(m_{n} c^{2}\right)^{2}+p_{n}^{2} c^{2}=\left(m_{\mathrm{e}} c^{2}\right)^{2}
$$

In this paper, Equation (17) will be derived more simply by using a method different from that used previously. The logic of Equations (11) to (15) is borrowed to accomplish that purpose.

Now, it is clear that the following equation holds.

$$
\left[m_{n} c^{2}+\left(m_{\mathrm{e}} c^{2}-m_{n} c^{2}\right)\right]^{2}=\left(m_{\mathrm{e}} c^{2}\right)^{2}
$$

Expanding and rearranging this equation, the following equation is obtained.

$$
\left(m_{n} c^{2}\right)^{2}+\left(m_{\mathrm{e}}+m_{n}\right)\left(m_{\mathrm{e}} c^{2}-m_{n} c^{2}\right) c^{2}=\left(m_{\mathrm{e}} c^{2}\right)^{2} .
$$

Next, the relativistic kinetic energy of the electron can be defined as follows by referring to Equation (15).

$$
K_{n}=m_{\mathrm{e}} c^{2}-m_{n} c^{2}=\frac{p_{n}^{2}}{m_{\mathrm{e}}+m_{n}} .
$$

From this,

$$
\left(m_{\mathrm{e}}+m_{n}\right)\left(m_{\mathrm{e}} c^{2}-m_{n} c^{2}\right)=p_{n}^{2} .
$$

Finally, Equation (19) matches Equation (17).

\section{Comparison of the Energy Levels of Bohr and This Paper}

The author has pointed out that the following relation is contained in Bohr's quantum condition [3] (Appendix).

$$
\frac{v_{n}}{c}=\frac{\alpha}{n} .
$$

Here, $\alpha$ is the following fine-structure constant.

$$
\alpha=\frac{e^{2}}{4 \pi \varepsilon_{0} \hbar c}=7.297352693 \times 10^{-3} .
$$

When both sides of Equation (22) are squared, and then multiplied by $m_{\mathrm{e}} / 2$,

$$
\frac{1}{2} \frac{m_{\mathrm{e}} v_{n}^{2}}{c^{2}}=\frac{1}{2} \frac{m_{\mathrm{e}} \alpha^{2}}{n^{2}}
$$

Hence,

$$
E_{\mathrm{BO}, n}=-\frac{1}{2} m_{\mathrm{e}} v_{n}^{2}=-\frac{\alpha^{2} m_{\mathrm{e}} c^{2}}{2 n^{2}}=-\frac{1}{2}\left(\frac{1}{4 \pi \varepsilon_{0}}\right)^{2} \frac{m_{\mathrm{e}} e^{4}}{\hbar^{2}} \cdot \frac{1}{n^{2}}, \quad n=1,2, \cdots .
$$

If Equation (22) is taken as a departure point, the energy levels of the hydrogen atom derived by Bohr can be derived immediately. Equation (22) has tremendous power. However, from a relativistic perspective, (1/2) $m_{\mathrm{e}} v_{n}^{2}$ is an approximation of the kinetic energy of the electron. Therefore, the energy in Equation (25) is also an approximation of the true value.

Now, the following formula for energy is obtained from Equation (2) [5] [6]. 


$$
m_{n}= \pm m_{\mathrm{e}}\left(\frac{n^{2}}{n^{2}+\alpha^{2}}\right)^{1 / 2}
$$

Equation (2) has a negative energy solution. However, here the energy of the hydrogen atom is regarded as a problem, and thus the positive solutions are used. In this case, the ordinary energy levels of the hydrogen atom are as follows.

$$
E_{n}=m_{n} c^{2}-m_{\mathrm{e}} c^{2}=m_{\mathrm{e}} c^{2}\left[\left(\frac{n^{2}}{n^{2}+\alpha^{2}}\right)^{1 / 2}-1\right]=m_{\mathrm{e}} c^{2}\left[\left(1+\frac{\alpha^{2}}{n^{2}}\right)^{-1 / 2}-1\right] .
$$

In contrast, the following is obtained when the energy levels of a hydrogen atom are derived from Sommerfeld's formula (2).

$$
E_{\mathrm{SO}, n}=m_{\mathrm{e}} c^{2}\left[1-\left(1-\frac{\alpha^{2}}{n^{2}}\right)^{-1 / 2}\right]
$$

However, when Equation (28) was derived, the $v / c$ in Equation (2) was replaced with $\alpha / n$. This replacement corresponds to quantization, for shifting from the continuous classical world to the world of quantum theory dominated by discontinuity.

Now, if a Taylor expansion is performed on the right side of Equations (27) and (28),

$$
\begin{gathered}
E_{n} \approx m_{\mathrm{e}} c^{2}\left[\left(1-\frac{\alpha^{2}}{2 n^{2}}+\frac{3 \alpha^{4}}{8 n^{4}}-\frac{5 \alpha^{6}}{16 n^{6}}\right)-1\right] \approx-m_{\mathrm{e}} c^{2}\left(\frac{\alpha^{2}}{2 n^{2}}-\frac{3 \alpha^{4}}{8 n^{4}}+\frac{5 \alpha^{6}}{16 n^{6}}\right) \\
E_{\mathrm{SO}, n} \approx m_{\mathrm{e}} c^{2}\left[1-\left(1+\frac{\alpha^{2}}{2 n^{2}}+\frac{3 \alpha^{4}}{8 n^{4}}+\frac{5}{16} \frac{\alpha^{6}}{n^{6}}\right)\right] \approx-m_{\mathrm{e}} c^{2}\left(\frac{\alpha^{2}}{2 n^{2}}+\frac{3 \alpha^{4}}{8 n^{4}}+\frac{5}{16} \frac{\alpha^{6}}{n^{6}}\right) .
\end{gathered}
$$

When these formulas are rewritten, the energy levels of the hydrogen atom are as follows.

$$
\begin{gathered}
E_{n} \approx-\frac{\alpha^{2}}{2 n^{2}} m_{\mathrm{e}} c^{2}\left(1-\frac{3 \alpha^{2}}{4 n^{2}}+\frac{5}{8} \frac{\alpha^{4}}{n^{4}}\right) . \\
E_{\mathrm{SO}, n} \approx-\frac{\alpha^{2}}{2 n^{2}} m_{\mathrm{e}} c^{2}\left(1+\frac{3 \alpha^{2}}{4 n^{2}}+\frac{5}{8} \frac{\alpha^{4}}{n^{4}}\right) .
\end{gathered}
$$

When $n=1$, the values of Equations (25), (27), and (28) are:

$$
\begin{gathered}
E_{\mathrm{BO}, 1}=-13.60569 \mathrm{eV} . \\
E_{1}=-13.60515 \mathrm{eV} . \\
E_{\mathrm{SO}, 1}=-13.60624 \mathrm{eV} .
\end{gathered}
$$

These energy differences are so minute they are difficult to experimentally discriminate.

\section{Discussion}

1) The potential energy of the electron was defined in Equation (6), but there is the following constraint on the values that potential energy can take [7]. 


$$
-m_{\mathrm{e}} c^{2} \leq V(r)<0
$$

On the other hand, potential energy is given by the following formula.

$$
E=-\frac{1}{4 \pi \varepsilon_{0}} \frac{e^{2}}{r}
$$

Hence, the radius $r$ when the value of potential energy is $-m_{\mathrm{e}} c^{2}$ is:

$$
r=\frac{e^{2}}{4 \pi \varepsilon_{0} m_{\mathrm{e}} c^{2}}=r_{\mathrm{e}} .
$$

Here, $r_{\mathrm{e}}$ is the classical electron radius. Even based on the classical perspective, it is evident that there are constraints on the distance at which the electron can approach the atomic nucleus.

Incidentally, in Equation (26), the electron has negative relativistic energy (that is, this electron has negative mass). However, if Equation (38) is taken into account, an electron at this energy level is not the electron constituting an ordinary hydrogen atom. An electron with negative energy exists near the atomic nucleus.

Here, if $r_{n}^{+}$is the orbital radius in an ordinary hydrogen atom, and $r_{n}^{-}$is the orbital radius of an electron at a negative energy level, then the ratio of the two is as follows [8].

$$
\frac{r_{1}^{-}}{r_{1}^{+}}=\frac{\left(1+\alpha^{2}\right)^{1 / 2}-1}{\left(1+\alpha^{2}\right)^{1 / 2}+1}=1.3312484168 \times 10^{-5} \approx \frac{1}{75120}
$$

$r_{n}^{-}$is far smaller than $r_{n}^{+}$. The author uses the name "dark hydrogen atom" for matter formed from one proton with positive mass and one electron with negative mass [9] [10]. A dark hydrogen atom is lighter than a hydrogen atom by about the mass of two electrons. However, the radius is extremely small, and thus it is possible to achieve a state of far greater density than ordinary matter. This is then observed as a tremendous mass.

The experiment that can demonstrate the existence of dark hydrogen is a triplet production experiment [8] [10].

In triplet production, $4 m_{\mathrm{e}} c^{2}(2.044 \mathrm{MeV})$ gamma rays are irradiated into a vacuum, and two electrons and one positron are produced. Changing the previous interpretation, the author regards one of the two produced electrons as an electron that formed dark hydrogen.

At present, it is thought that stars and galaxies were created in the center of dark halos, i.e., masses of dark matter. The idea is that, due to the tremendous gravity of a dark halo, surrounding atoms and matter are drawn in, and stars and galaxies are formed. At present, dark matter is believed to be a material which does not emit or absorb light. However, the dark hydrogen atoms and other dark atoms presented in this paper do emit and absorb photons. However, the photons emitted from these dark atoms are not energetically distinguishable from photons emitted from ordinary atoms. Normal hydrogen atoms can be produced if dark hydrogen atoms are irradiated with gamma rays of about 
$2 m_{\mathrm{e}} c^{2}(1.022 \mathrm{MeV})$. That is, in the model presented in this paper, dark matter is turned into ordinary matter simply through the absorption of energy.

2) When Dirac derived the relativistic wave equation, his departure point was Equation (1). However, when deriving a wave equation applicable to the electron in a hydrogen atom, the departure point should be Equation (17). The author has derived a relativistic wave equation to replace the Dirac equation from this perspective [6] [11] [12].

\section{Conclusions}

The relationship in the STR, which holds for an isolated system in free space, is given by the following formula.

$$
\left(m_{0} c^{2}\right)^{2}+p^{2} c^{2}=\left(m c^{2}\right)^{2} .
$$

In contrast, the author has previously derived an energy-momentum relationship applicable to an electron in a hydrogen atom where there is potential energy. This is the following formula.

$$
\left(m_{n} c^{2}\right)^{2}+p_{n}^{2} c^{2}=\left(m_{\mathrm{e}} c^{2}\right)^{2}
$$

In this paper, Equation (41) was derived using a method simpler than that used before. Since this single formula was logically derived using two different methods, the author believes the correctness of Equation (41) has been proven.

From Equation (41), it is possible to predict more exact values than the energy levels of a hydrogen atom derived from Bohr's classical quantum theory. Also, it can be predicted that, when Equation (41) is solved, there is an $n=0$ energy level in a hydrogen atom, and that a state exists where relativistic energy levels are negative.

Matter with negative energy levels is formed from a proton with positive mass and an electron with negative mass. The author has given the name "dark hydrogen" to this matter. This matter is a strong candidate for dark matter, whose true nature is currently unknown.

However, the dark matter problem may be solved also through the extended theories of gravity [13].

\section{Acknowledgments}

I would like to express my thanks to the staff at ACN Translation Services for their translation assistance.

\section{Conflicts of Interest}

The authors declare no conflicts of interest regarding the publication of this paper.

\section{References}

[1] Sommerfeld, A. (1923) Atomic Structure and Spectral Lines. Methuen \& Co. Ltd., 
London, 528.

[2] Suto, K. (2009) True Nature of Potential Energy of a Hydrogen Atom. Physics Essays, 22, 135-139. https://doi.org/10.4006/1.3092779

[3] Suto, K. (2019) The Relationship Enfolded in Bohr's Quantum Condition and a Previously Unknown Formula for Kinetic Energy. Applied Physics Research, 11, 19-34. https://doi.org/10.5539/apr.v11n1p19

[4] Suto, K. (2011) An Energy-Momentum Relationship for a Bound Electron inside a Hydrogen Atom. Physics Essays, 24, 301-307. https://doi.org/10.4006/1.3583810

[5] Suto, K. (2014) $n=0$ Energy Level Present in the Hydrogen Atom. Applied Physics Research, 6, 109-115. https://doi.org/10.5539/apr.v6n5p109

[6] Suto, K. (2014) Previously Unknown Ultra-Low Energy Level of the Hydrogen Atom Whose Existence Can Be Predicted. Applied Physics Research, 6, 64-73. https://doi.org/10.5539/apr.v6n6p64

[7] Suto, K. (2018) Potential Energy of the Electron in a Hydrogen Atom and a Model of a Virtual Particle Pair Constituting the Vacuum. Applied Physics Research, 10, 93-101. https://doi.org/10.5539/apr.v10n4p93

[8] Suto, K. (2017) Region of Dark Matter Present in the Hydrogen Atom. Journal of Physical Mathematics, 8, Article ID: 1000252.

[9] Suto, K. (2015) Presentation of Strong Candidates for Dark Matter. Global Journal of Science Frontier Research: A, 15, 1-6.

[10] Suto, K. (2017) Presentation of Dark Matter Candidates. Applied Physics Research, 9, 70-76. https://doi.org/10.5539/apr.v9n1p70

[11] Suto, K. (2011) Alternative Formulation of Relativistic Quantum Mechanics. Physics Essays, 24, 598-602. https://doi.org/10.4006/1.3659280

[12] Suto, K. (2018) Derivation of a Relativistic Wave Equation More Profound than Dirac's Relativistic Wave Equation. Applied Physics Research, 10, 102-108. https://doi.org/10.5539/apr.v10n6p102

[13] Corda, C. (2009) Interferometric Detection of Gravitational Waves: The Definitive Test for General Relativity. International Journal of Modern Physics D, 18, 2275-2282. https://doi.org/10.1142/S0218271809015904 


\section{Appendix}

Bohr thought the following quantum condition was necessary to find the energy levels of the hydrogen atom.

$$
m_{\mathrm{e}} v_{n} \cdot 2 \pi r_{n}=2 \pi n \hbar
$$

Here,

$$
\hbar=\frac{h}{2 \pi}=\frac{m_{\mathrm{e}} c \lambda_{\mathrm{C}}}{2 \pi}
$$

Next, when $r_{\mathrm{e}} / \alpha$ is found,

$$
\frac{r_{\mathrm{e}}}{\alpha}=\frac{e^{2}}{4 \pi \varepsilon_{0} m_{\mathrm{e}} c^{2}} \frac{4 \pi \varepsilon_{0} \hbar c}{e^{2}}=\frac{\lambda_{\mathrm{C}}}{2 \pi} .
$$

Here, $r_{\mathrm{e}}$ is the classical electron radius and $\alpha$ is the fine structure constant. Each of these can be written as follows.

$$
\begin{gathered}
r_{\mathrm{e}}=\frac{e^{2}}{4 \pi \varepsilon_{0} m_{\mathrm{e}} c^{2}} . \\
\alpha=\frac{e^{2}}{4 \pi \varepsilon_{0} \hbar c} .
\end{gathered}
$$

Also, the radius of the electron orbital derived by Bohr can be rewritten as follows.

$$
r_{n}=4 \pi \varepsilon_{0} \frac{\hbar^{2}}{m e^{2}} n^{2}=\frac{e^{2}}{4 \pi \varepsilon_{0} m_{\mathrm{e}} c^{2}}\left(\frac{4 \pi \varepsilon_{0} \hbar c}{e^{2}}\right)^{2} n^{2}=\frac{r_{\mathrm{e}}}{\alpha^{2}} n^{2}=\frac{\lambda_{\mathrm{C}}}{2 \pi \alpha} n^{2} .
$$

If the value of $r_{n}$ in Equation (A6) is substituted into Equation (A1),

$$
m_{\mathrm{e}} v_{n} \frac{\lambda_{\mathrm{C}}}{\alpha} n^{2}=m_{\mathrm{e}} c \lambda_{\mathrm{C}} n
$$

Using this,

$$
\frac{v_{n}}{c}=\frac{\alpha}{n}
$$

Equation (A8) is the core part of Bohr's quantum condition (A1). This condition is non-relativistically correct, but relativistically it is incorrect. However, Equation (A8) is also valid when deriving a relativistic formula.

Next, let's try to derive Equation (26) from Equation (A8). If both sides of Equation (A8) are first squared, and then both sides are multiplied by $m_{n}^{2} /\left(m_{\mathrm{e}}+m_{n}\right)$.

$$
\frac{m_{n}^{2} v_{n}^{2}}{m_{\mathrm{e}}+m_{n}}=\frac{\alpha^{2} c^{2}}{n^{2}} \frac{m_{n}^{2}}{m_{\mathrm{e}}+m_{n}}
$$

Here, the left side of Equation (A9) is the kinetic energy of the electron, and thus the energy level is:

$$
E_{n}=-K_{n}=-\frac{m_{n}^{2} v_{n}^{2}}{m_{\mathrm{e}}+m_{n}}=-\frac{\alpha^{2} c^{2}}{n^{2}} \frac{m_{n}^{2}}{m_{\mathrm{e}}+m_{n}} .
$$


Next, if Equation (25) is taken into account, the right side of Equation (A10) is as follows.

$$
E_{n}=-\frac{\alpha^{2} c^{2}}{n^{2}} \times\left(\frac{n^{2} m_{\mathrm{e}}^{2}}{n^{2}+\alpha^{2}}\right) \times \frac{1}{m_{\mathrm{e}}\left[1+\left(\frac{n^{2}}{n^{2}+\alpha^{2}}\right)^{1 / 2}\right]}
$$

Next, the numerator and denominator of Equation (A11) are multiplied by:

$$
1-\left(\frac{n^{2}}{n^{2}+\alpha^{2}}\right)^{1 / 2}
$$

When this is done, Equation (A11) is as follows.

$$
\begin{aligned}
E_{n} & =-\frac{\alpha^{2}}{n^{2}}\left(\frac{n^{2}}{n^{2}+\alpha^{2}}\right) m_{\mathrm{e}} c^{2} \times\left(\frac{n^{2}+\alpha^{2}}{\alpha^{2}}\right)\left[1-\left(\frac{n^{2}}{n^{2}+\alpha^{2}}\right)^{1 / 2}\right] \\
& =m_{\mathrm{e}} c^{2}\left[\left(\frac{n^{2}}{n^{2}+\alpha^{2}}\right)^{1 / 2}-1\right] \\
& =m_{\mathrm{e}} c^{2}\left[\left(1+\frac{\alpha^{2}}{n^{2}}\right)^{-1 / 2}-1\right] .
\end{aligned}
$$

Taking Equation (A8) as a departure point, it was possible to derive Equation (26) for the energy levels in a hydrogen atom. 